\title{
geneHummus: an R package to define gene families and their expression in legumes and beyond
}

\author{
Jose V. Die ${ }^{1,2}$, Moamen M. Elmassry ${ }^{2,3}$, Kimberly H. LeBlanc ${ }^{4}$, Olaitan I. Awe ${ }^{2,5}$, Allissa Dillman² and Ben Busby ${ }^{2 *}$ (D)
}

\begin{abstract}
Background: During the last decade, plant biotechnological laboratories have sparked a monumental revolution with the rapid development of next sequencing technologies at affordable prices. Soon, these sequencing technologies and assembling of whole genomes will extend beyond the plant computational biologists and become commonplace within the plant biology disciplines. The current availability of large-scale genomic resources for non-traditional plant model systems (the so-called 'orphan crops') is enabling the construction of high-density integrated physical and genetic linkage maps with potential applications in plant breeding. The newly available fully sequenced plant genomes represent an incredible opportunity for comparative analyses that may reveal new aspects of genome biology and evolution. The analysis of the expansion and evolution of gene families across species is a common approach to infer biological functions. To date, the extent and role of gene families in plants has only been partially addressed and many gene families remain to be investigated. Manual identification of gene families is highly time-consuming and laborious, requiring an iterative process of manual and computational analysis to identify members of a given family, typically combining numerous BLAST searches and manually cleaning data. Due to the increasing abundance of genome sequences and the agronomical interest in plant gene families, the field needs a clear, automated annotation tool.
\end{abstract}

Results: Here, we present the geneHummus package, an R-based pipeline for the identification and characterization of plant gene families. The impact of this pipeline comes from a reduction in hands-on annotation time combined with high specificity and sensitivity in extracting only proteins from the RefSeq database and providing the conserved domain architectures based on SPARCLE. As a case study we focused on the auxin receptor factors gene (ARF) family in Cicer arietinum (chickpea) and other legumes.

Conclusion: We anticipate that our pipeline should be suitable for any taxonomic plant family, and likely other gene families, vastly improving the speed and ease of genomic data processing.

Keywords: Bioinformatics, Genome annotation, Gene family, Plant breeding, Rstat, Pipeline, Phylogenetic tree, RefSeq, SPARCLE

\section{Background}

By using next-generation sequencing (NGS) technology, researchers have massively increased the number of nucleotide sequences deposited in public databases [1]. This had revolutionized numerous fields including the plant sciences (Fig. 1). However, a bottleneck in the field of plant sciences is the annotation of the protein

\footnotetext{
* Correspondence: Ben.Busby@nih.gov

${ }^{2}$ National Center for Biotechnology Information, National Library of Medicine, National Institutes of Health, 8600 Rockville Pike, Bethesda, MD 20894, USA Full list of author information is available at the end of the article
}

sequences and the characterization of their functions. Identifying the function of important proteins can be used to improve agronomic performance, like altering resistance or tolerance of plants to specific environmental stressors such as drought or heat. One approach to infer the function of an unknown protein is to identify conserved sequences among proteins with known function, which can be useful to the extent that homology can imply conserved biochemical function [2].

The plant hormone auxin (indole-3-acetic acid) is a key regulator of virtually every aspect of plant growth

(c) The Author(s). 2019 Open Access This article is distributed under the terms of the Creative Commons Attribution 4.0 International License (http://creativecommons.org/licenses/by/4.0/), which permits unrestricted use, distribution, and reproduction in any medium, provided you give appropriate credit to the original author(s) and the source, provide a link to the Creative Commons license, and indicate if changes were made. The Creative Commons Public Domain Dedication waiver (http://creativecommons.org/publicdomain/zero/1.0/) applies to the data made available in this article, unless otherwise stated. 


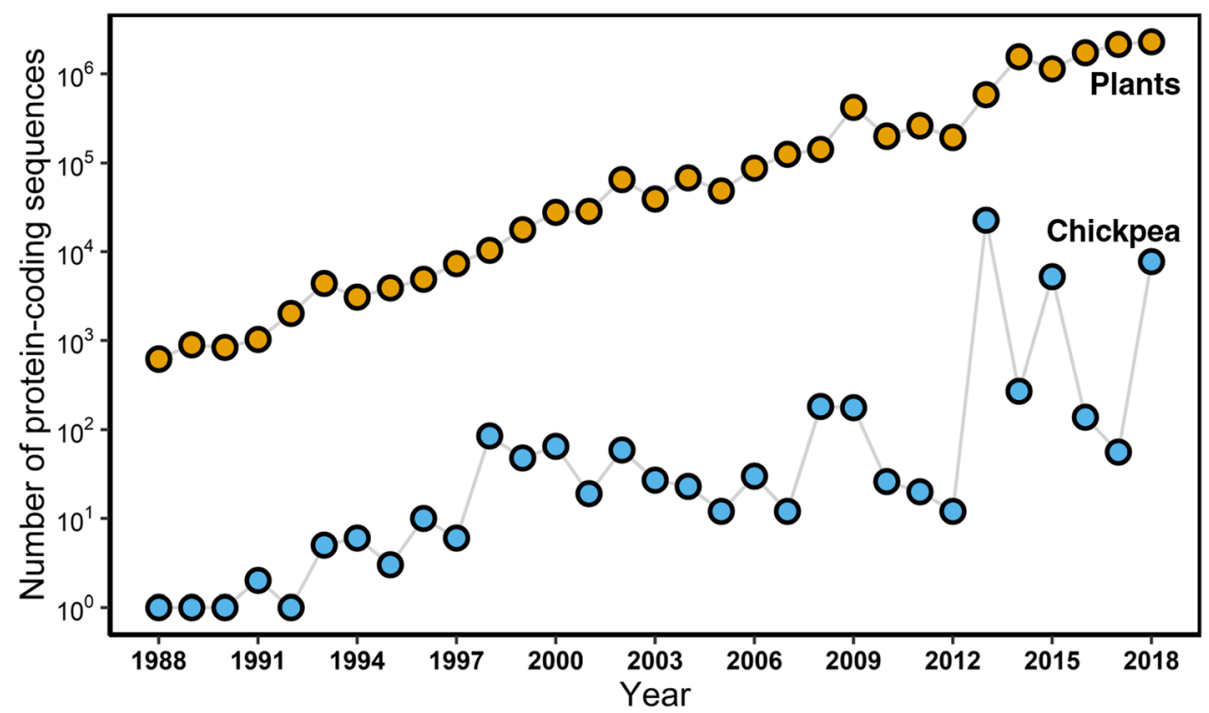

Fig. 1 Statistics on the number of protein-coding sequences deposited in NCBI every year. Number of plants and chickpea protein-coding sequences deposited in NCBI over the past 3 decades

and development [3]. As a central role of the auxin-signaling pathway, the auxin response factor (ARF) multigene family is present in all major divisions of land plants [4]. Considering the important role of ARF family members as regulators of plant growth and developmental processes, in the last few years there has been a considerable interest in studying the ARF family in both annual herbaceous plants $[5,6]$ and woody perennials $[7,8]$. Characterization of ARF typically gives insights into the genomic structures [9], loci distribution across the genomes [10], sequence homology [11], phylogenetic history [12], and gene expression patterns [13] during development and/or biotic/abiotic stress. Efficient characterization of ARF family members could vastly improve both the number of useful genomic targets that could be identified to improve agronomic performance, and the speed with which they are characterized. Doing so within the legume family could have a dramatic impact on the availability of nutritious food and on ecosystem resilience [14].

Currently, the identification and characterization of plant gene families is laborious and time-consuming. It requires an iterative process of computational analysis to identify the gene family members of a given family. This process is based mainly on Basic Local Alignment Search Tool (BLAST) [15] searches interspersed with manual curation and pruning (Additional file 1: Figure S1). Due to the increasing number of sequences and the agronomical interest in plant gene families, this process could benefit from an automated tool. Although, numerous bioinformatics tools have been already developed to identify homologous proteins, these tools are largely restricted to static databases of fully sequenced animal genomes, and thus a tool that could work for plant genomes is needed.

To meet this need, we developed geneHummus, a novel $\mathrm{R}$ package that efficiently identifies members (accessions) of a plant gene family. By querying the SPARCLE and RefSeq databases, geneHummus can quickly isolate architecture identifiers from whole or draft genomes across taxonomic kingdoms, and update as new sequences are accessioned. In addition, geneHummus simplifies downstream analysis such as phylogenetic constructions and gene expression profiles. As a case study we focused on the auxin receptor factors gene (ARF) family in Cicer arietinum (chickpea) and other legumes, having previously manually annotated the ARF family in the chickpea genome [5], therefore having a gold standard dataset to which to compare our pipeline results. In our case study on the ARF gene family, the functionality of geneHummus allows an integrated workflow with phylogeny and expression profiles. We anticipate that our pipeline should be suitable for the study of any plant gene family, and likely other gene taxonomic families, vastly improving the speed and ease of genomic data processing.

\section{Implementation}

geneHummus is implemented as an $\mathrm{R}$ package and requires a minimal set of dependencies (dplyr [16], stringr [17], rentrez [18], httr, utils and curl packages) which are automatically downloaded from the CRAN repository [19] as needed. In this section, we describe the implementation of geneHummus in detail. There is also additional documentation describing the ARF case study available from our Github repository [20]. This pipeline was designed for a biologist end-user with a minimal 
amount of programming experience, using open source and free software (R, NCBI tools) to guide the user through the identification, characterization and expression analysis of gene families. Plant gene families are characterized by common protein structure. The structure that defines a given family is known from literature. For example, the hidden Markov model (HMM) profiles of the ARF gene family are the B3 DNA binding domain (B3), AUX_RESP, and AUX/IAA, which correspond with the conserved domains Pfam 02362, Pfam 06507, and Pfam 02309. The pipeline begins by defining the conserved domains accession numbers as a query against the SPARCLE database at the NCBI (Fig. 2). Then, you can get the SPARCLE architecture identifiers (ids) for each conserved domain and extract, or filter, only those architectures that characterize the ARF gene family. Next, the protein electronic ids for each candidate ARF architecture are retrieved. Depending on your dataset this step may take from seconds to $3-5 \mathrm{~min}$. Note that if you have a very long list of protein ids, you may receive a 414 error when you try to interact with the NCBI Eutilities. geneHummus subsets the elements (up to 300 ids per list), so the functions can work properly. The retrieved protein ids are filtered by the taxonomy ids of interest [21], which in this case study were the legumes ids. genehummus returns only the protein ids hosted by the RefSeq database. Finally, the electronic ids are converted to protein accessions. At this point we have likely identified the whole set of ARF protein accessions from the legume family. Downloading the amino acid sequences from the accessions is straightforward. Once these sequences are downloaded, the relevant information may be used for phylogenetic and expression studies.

\section{Downstream analyses outside of the geneHummus $\mathbf{R}$ package Phylogenetic analysis}

Multiple protein sequence alignments (MSAs) were performed on the conserved Pfam domains [Pfam 02309: AUX/IAA family; Pfam 06507: ARF (AUX_RESP); Pfam 02362: B3 DNA binding domain (B3)] for the whole dataset. Multiple alignments were performed with MAFFT version 7.402 [22] using standard methods (FFT-NS-i) and the following parameters: mafft --thread 10 --threadtb 5 -- threadit 0 --reorder --leavegappyregion. A recent online version of the software is available as well [23]. A NJ tree was conducted using the JTT substitution model, 500 replicates of bootstrap, and pairwise detection of gaps. Two representative of gymnosperms (Picea sitchensis and Pinus pinaster) were included as outgroup species.

\section{Expression analysis}

Using 1 single gene-model per locus, we created a BLAST database with the 24 ARF genes from the

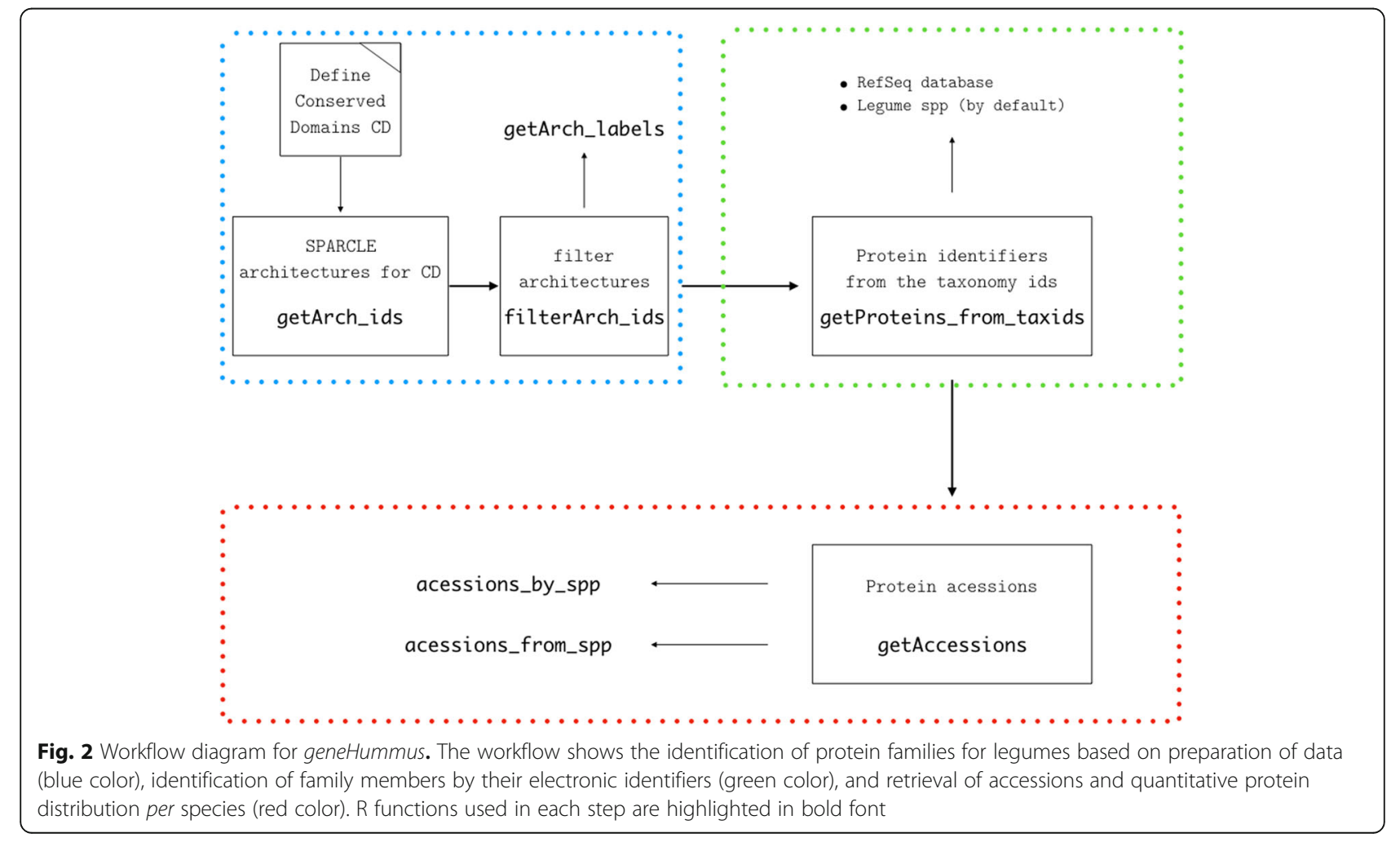


chickpea genome. Using Magic-BLAST [24], we studied the frequency of the ARF family in root tissues of two genotypes under drought stress and control conditions across 4 publicly available SRA libraries with the following parameters: alignment score $=125 \mathrm{bp}$, alignment identity $\geq 99 \%$ and read abundance in the four SRA libraries -- a list can be found at [25]. A normalization factor was estimated for each SRA library by dividing the average SRA size by the corresponding SRA size. The normalization factor was applied to each read to give normalized counts.

\section{Results and discussion}

\section{External validation of data}

Numerous approaches have been developed to predict the function of different proteins. The Subfamily Protein Architecture Labeling Engine (SPARCLE; [26]), a recently developed resource by National Center for Biotechnology Information (NCBI), is one such approach. SPARCLE can help functional characterization of protein sequences by grouping them according to their characteristic domain architecture. We searched the SPARCLE database to obtain the whole set of molecular architectures based on the conserved domains that define the ARF gene family. Then, we filtered the data from SPARCLE to select the taxonomic group for the legume family (Fabaceae), and the source database (RefSeq; [27]). After these filters, we obtained over 560 different ARF legume proteins encoded by $\sim 330$ gene loci (Fig. 3). After separating these results by species, our pipeline identified 24 ARF proteins in the chickpea genome (Cicer arietinum), reproducing the results obtained previously with an

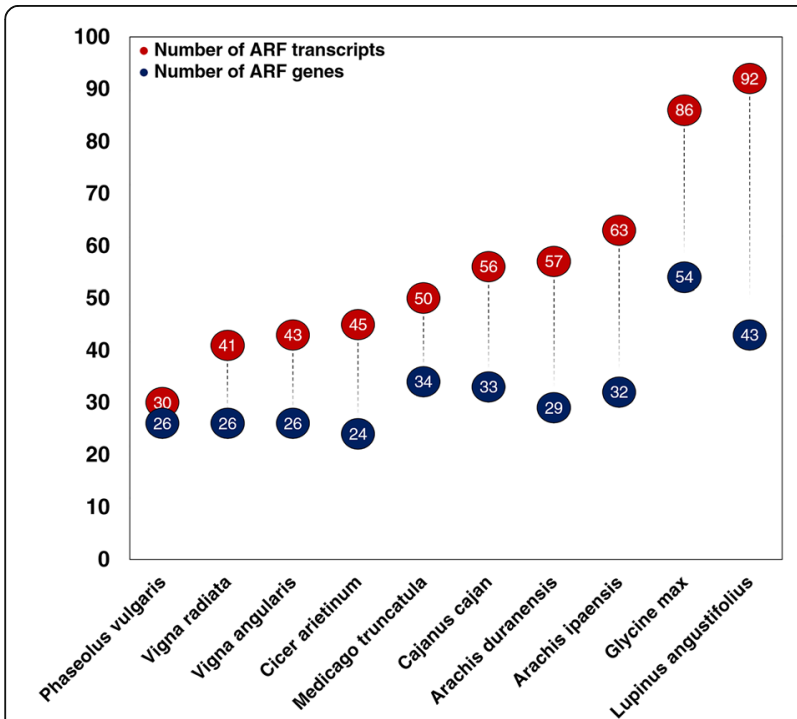

Fig. 3 Total number of ARF sequences identified by CDART in legumes. Blue circles shows the number of ARF genes in each species of legumes, while red circles show the number of ARF transcripts iterative exhaustive BLAST search [5]. These results validate the geneHummus approach, which provides an automated way to produce these results in less than $6 \mathrm{~min}$, as opposed to the exhaustive BLAST method which required significant manual curation and over 6 months of work. In addition, the geneHummus pipeline also returned the number of ARF proteins in 9 other legume species in this 6 min processing window (Fig. 3). Interestingly, the number of ARF proteins is similar in different species within the same genus (Arachis duranesis and Arachis ipaensis; Vigna angularis and Vigna radiata), as may be expected. In addition, species known to possess a high number of expanded paralogous genes due to whole-genome duplications events, such as Glycine and Lupinus lineages [28], showed the highest values both in the number of transcripts and number of ARF loci. We found that geneHummus is specific and sensitive by identifying the same sequences previously reported in other legume studies characterizing the ARF family through exhaustive searches, such as $M$. truncatula or soybean $[6,29]$. But we also found more ARF accessions than had ever previously been reported, as geneHummus uses the latest updated RefSeq database version available at GenBank (Table 1). All of these results increase confidence in the validity of our approach. To find the ARFs in the different legume species using our pipeline, we developed an interactive shiny application to access this data [30]. After running the geneHummus pipeline, researchers interested in loading the relevant table for their gene families and taxa of interest can clone and modify [31] to easily share their results.

\section{Comparison with existing software}

To date, a number of tools and pipelines are available to analyze sequences based on gene families. These tools are excellent for the identification of sequences in whole animal genomes; however, most of them cannot be applied to plant genomes or draft genomes, and have other limitations based on the databases they query and the software they use (see Table 2).

geneHummus is a flexible tool that can work with almost any gene family and almost any plant species. HomoloGene [37] is an automated system for detecting homologs among 21 completely sequenced eukaryotic genomes; however, it is not flexible enough to be applied on draft genomes. MultiMSOAR [38, 39] and OrthoMCL $[40,41]$ are other tools that were developed to find ortho$\log$ groups among different genomes. These tools are limited to the software version and genomes included, which does not include the chickpea genome or other legume species. Ensembl plants [42] also provides programming tools to extract target genes families, but is similarly restricted to a limited number of genomes and the sequences that are hosted by the browser. While these 
Table 1 Genome-wide identification of ARF gene family in plant species using exhaustive BLAST searches and geneHummus approaches

\begin{tabular}{llll}
\hline Species & Genome-wide analysis & geneHummus & Number of transcripts \\
\cline { 3 - 4 } & Number of genes & 22 & 44 \\
\hline Arabidopsis thaliana & $22[32]^{\text {a }}$ & 17 & 27 \\
Prunus persica & $18[8]$ & 34 & 50 \\
Medicago truncatula & $24[6]$ & 54 & 86 \\
Glycine max & $51[29]$ & 17 & 27 \\
Eucalyptus grandis & $17[33]$ & 34 & 48 \\
Brassica rapa & $31[34]$ & 20 & 38 \\
Solanum lycopersicum & $17[35]$ & 19 & 31 \\
Vitis vinifera & $19[36]$ & & \\
\hline
\end{tabular}

${ }^{a}$ Okushima et al. reported 22 full-length ARF genes and one pseudo-gene

tools are excellent for their intended purposes, they cannot meet the needs of plant geneticists interested in identifying conserved protein architectures.

geneHuтmus goes beyond existing tools in several ways. It identifies sequences for performing further analysis by searching for protein architectures from NCBI data, and then retrieving the gene family without requiring downloading of the gene or protein sequences. It uses the latest updated reference sequences, and is therefore more comprehensive in both the breadth of species and families covered for whole and partial genomes, as well as more accurate than static databases. Although geneHummus requires the end user to have a minimal familiarity with the $\mathrm{R}$ programming language, through the sequential call of four functions, the pipeline identifies the given gene family in a user-friendly and rapid environment. Provided that sequences are hosted by GenBank, the major advantage of geneHummus is that the user can apply it on the fly for any genome and it can be customized to be suitable for other agronomically important taxonomic families beyond legumes. For example, when installing the package, the user has access to several objects that contain the taxonomy ids for the families Brassicaceae, Cucurbitaceae, Rosaceae, and Solanaceae. This can be also customized for other families, which in practical terms makes geneHummus a useful tool for the plant research community. A summary of the above statements can be seen in Table 2 .

\section{Phylogenetic analysis}

Numerous downstream analyses and applications can be performed on geneHummus results. One example is phylogenetic analysis. Based on the conserved domains of ARF proteins, we explored and depicted the sequence relationships between the whole dataset. Two ARF proteins from the gymnosperm lineage were included as outgroup species. Gymnosperms have been resolved as

Table 2 Currently available ortholog identification tools

\begin{tabular}{|c|c|c|}
\hline Tool & Purpose and Features & Platform \\
\hline HomoloGene & $\begin{array}{l}\text { - Constructs orthologous groups from the complete gene sets of } 21 \\
\text { eukaryotic species } \\
\text { - Includes only species with a complete genome or at least 10,000 } \\
\text { UniGene entries }\end{array}$ & - Web interface \\
\hline MultiMSOAR & $\begin{array}{l}\text { - Identifies ortholog groups among multiple genomes } \\
\text { - Genome should be closely related }\end{array}$ & - Linux \\
\hline OrthoMCL & - Groups proteins into ortholog groups based on their sequence similarity & $\begin{array}{l}\text { - Galaxy server } \\
\text { - Linux }\end{array}$ \\
\hline GeneSeqToFamily & $\begin{array}{l}\text { - Finds orthologous genes and their corresponding gene families using the } \\
\text { Ensembl Compara GeneTrees pipeline }\end{array}$ & - Galaxy server \\
\hline OrthoFinder & - Identifies orthologous protein sequence families & - Linux \\
\hline Ensembl Plants & $\begin{array}{l}\text { - Utilizes reference genome sequences as a framework to integrate variant, } \\
\text { functional, expression, marker, and comparative data for a number of plant species } \\
\text { - Ensembl plants does not include most legumes }\end{array}$ & $\begin{array}{l}\text { - Web interface } \\
\text { - API }\end{array}$ \\
\hline geneHummus & $\begin{array}{l}\text { - Uses the Refseq Database, which is dynamically growing and manually curated } \\
\text { - Sequence data is streamed within cloud or local infrastructure so it doesn't } \\
\text { require downloading of genomic or protein sequences }\end{array}$ & $\begin{array}{l}\text { - R } \\
\text { - Linux }\end{array}$ \\
\hline
\end{tabular}


the sister group of angiosperms. They diverged from their most recent common ancestor $\sim 310$ million years ago [43]. The phylogenetic distribution of the protein sequences revealed that all ARF sequences fall into two major groups (I and II) with well-supported bootstrap values (Fig. 4). The group I is the most numerous and may be further subdivided into clusters containing orthologs of the Arabidopsis sequences defining the well-known clades AtARF3/4-like, ARF12-like, ARF10/ 16-like, and ARF17-like [32]. Group II contain the cluster AtARF5-like. A second cluster in group II did not contain any Arabidopsis ortholog (data not shown) implying that this clade was derived through a long-term evolution for conserved functions across legume plant species. We labeled as sister pairs those proteins clustered together based on high bootstrap values (>65\%). Related to sister pairs involving chickpea, the phylogeny structures 8 sister pairs (seven pairs of $C$. arietinum- $M$. truncatula and one pair of $C$. arietinum-L. angustifolius). We did not observe any sister pair between two chickpea ARF proteins. This is an interesting evolutionary pattern. Chickpea diverged from M. truncatula $\sim 10-20$ million years ago [14]. Lack of chickpea sister pairs suggests that recent duplications (after chickpea and Medicago separated) have played a very limited role, if any, in the expansion of the ARF chickpea family, or that duplicated proteins did not change much since both species shared a common ancestor. Both hypothesis are plausible as well.

In addition, within the AtARF12-like group we observed a distinct clade made of 4 proteins based on bootstrap value, belonging to the ancestors of the cultivated peanut [44]. This suggests that these are orthologs of an ancestral gene emerged after the speciation of Arachis genus (Additional file 2: Figure S2). We are looking forward to the peanut genome becoming public so we can validate these results.

\section{Expression profiles for gene discovery}

Another useful application that geneHuтmus results could be used for is identifying genes of interest in transcriptomic experiments and their role in responding to environmental perturbations. We used the genes identified by geneHummus as a reference database to study their expression in drought conditions using freely available SRA data and the Magic-BLAST tool [24]. We identified datasets in the SRA that isolated sequences from the root of the chickpea plant grown in either drought or control conditions, and was identified as belonging to either a tolerant/drought or susceptible/ drought strain. Upon analysis, we identified 3 transcripts that were differentially expressed in the drought-tolerant strain in drought conditions as opposed to the drought-susceptible strain (Fig. 5). This ARFs could be important targets for the genetic improvement of chickpea via conventional breeding or biotechnological approaches.

\section{Conclusions}

Applying the geneHummus pipeline, we characterized the structure and phylogeny of the whole ARF proteins dataset in the legume family. As a case study, we also

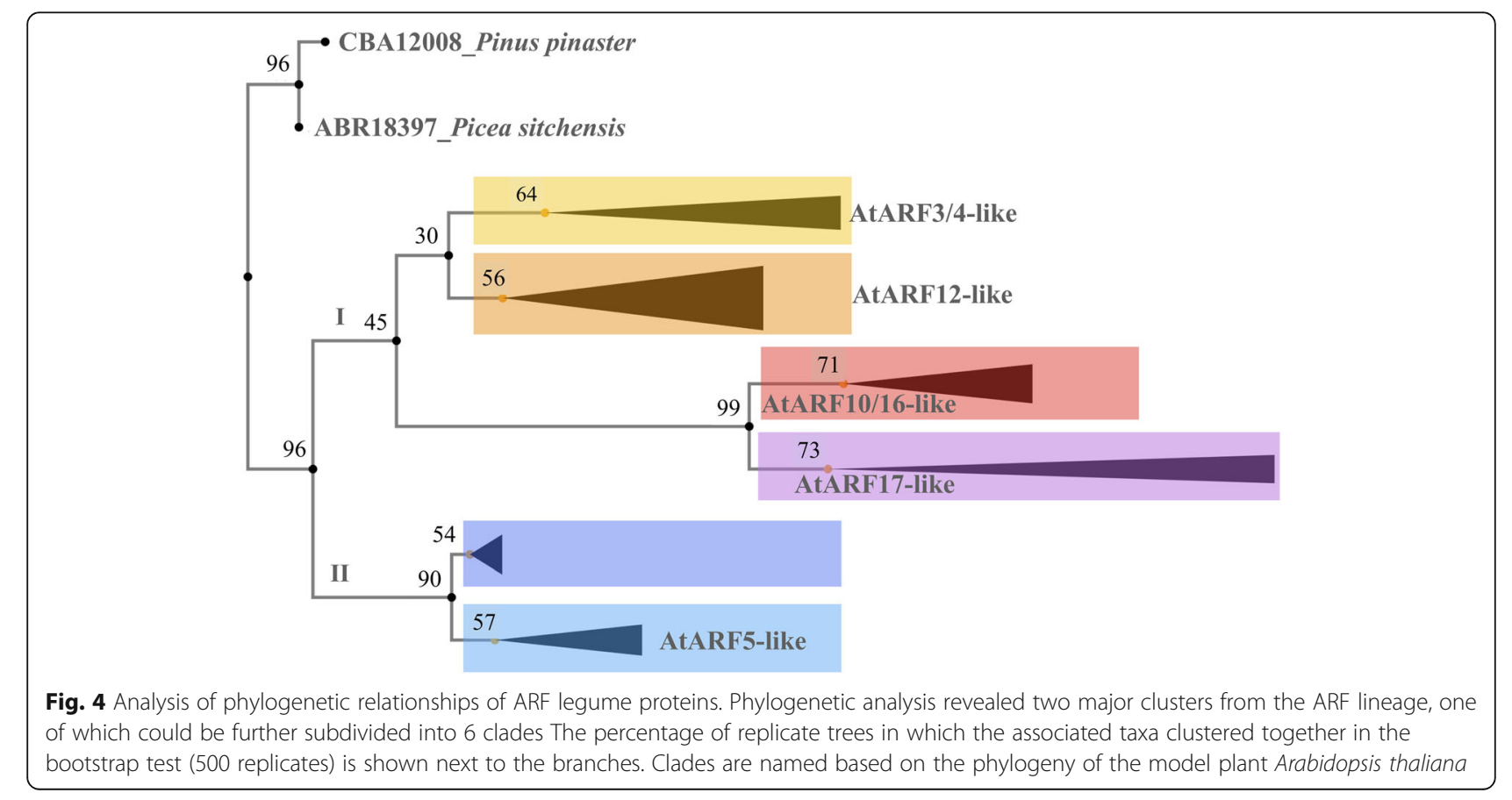




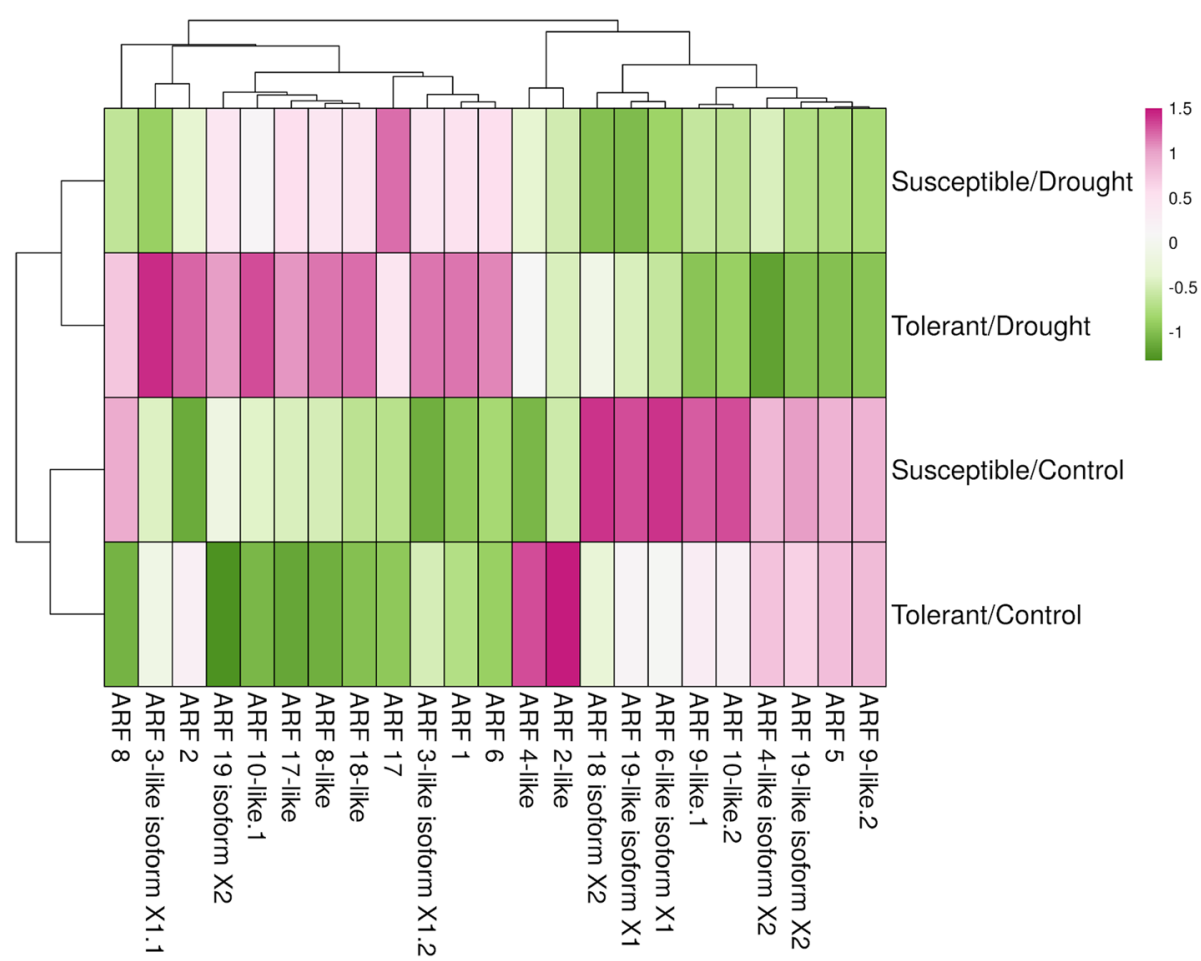

Fig. 5 Differential abundance of ARF gene loci in Cicer arietinum under drought or normal conditions. Drought samples are clustered on the top, while control samples are clustered on the bottom

characterized the expression profile of the gene family in Cicer arietinum. The utility of this pipeline stems from a reduction in hands-on annotation time combined with high specificity and sensitivity in extracting proteins from the RefSeq database and providing interaction with the suite of other NCBI tools such as the conserved domain architectures based on the recently developed NCBI resources SPARCLE and the aligner MagicBLAST. geneHummus is a powerful tool for the identification of gene family sequences that could be used in phylogenetic analysis. Our results indicates that most proteins are very well conserved across genera, with abundant multi-species clades. This suggests that these proteins are involved in common basic cellular actions. This orthology information could be used to infer the function of a previously uncharacterized protein in a given species based on the known function of the protein in another genera. This is a particularly strong approach for comparative genomics. Once the sequences have been identified, given the ability of available SRA libraries for a number of tissues and conditions, the user can get the most out of the pipeline by using MagicBLAST-based differential expression analysis to identify genes of interest for certain conditions. This tool will help investigators discover genes, and has particular applicability to plant breeding programs, among other applications. Our pipeline has been suitable for ARF family detection on all plant Genomes tested, and should be suitable for other gene families, vastly improving the speed and ease of genomic data processing.

\section{Availability and requirements}

Project name: geneHummus.

Project home page: https://github.com/NCBI-Hackathons/GeneHummus

Operating system(s): Platform independent.

Programming language: $R$.

Other requirements: R 3.5.0 or higher.

License: MIT license.

Any restrictions to use by non-academics: None.

\section{Additional files}

Additional file 1: Figure S1. Approaches for identification of gene families. a. Exhaustive identification based on BLAST searches. Sequences from a close-related species are used as queries using BLAST to identify the corresponding gene members in a target genome. For validation, sequences from a second model organism are also commonly used as queries following the same procedure. The hidden Markov model profiles of the gene family is used to confirm the identity of the candidate genes. b. Automatic pipeline implemented in geneHummus. The defining conserved domains of the gene family are used to parse the SPARCLE database and retrieve sequences grouped by a given protein architecture. (JPEG $199 \mathrm{~kb}$ )

Additional file 2: Figure S2. Cluster from clade AtARF12-like containing Arachis specific ARFs. (TIFF $1501 \mathrm{~kb}$ ) 


\section{Abbreviations}

ARF: Auxin receptor factor; BLAST: Basic Local Alignment Search Tool; HMM: Hidden Markov model; MSA: Multiple sequence alignment; NCBI: National Center for Biotechnology Information; NGS: Next-generation sequencing; SPARCLE: Subfamily Protein Architecture Labeling Engine; SRA: Sequence Read Archive

\section{Acknowledgements}

We would like to thank Jose Blanca (COMAV, Valencia) for his comments on functions. We would like to thank Greg Boratyn, Aaron Marchler-Bauer and Lewis Geer from NCBI Magic-BLAST and SPARCLE, and Jonathan Kans, the author of EDirect, and Steve Tsang for helpful discussions. We would also like to thank Dan Smith, the lead administrator of the NCBI visiting bioinformatics program.

\section{Authors' contribution}

JVD and MME participated in the design of the study, performed the bioinformatics and statistical analyses, generated figures and drafted the manuscript. KHL made substantial contributions to the bioinformatics analysis and the manuscript draft. OIA made contributions to the bioinformatics analysis and the manuscript draft and editing. AD made contributions to the bioinformatics analysis. BB supervised the project and contributed to the manuscript draft. All authors read and approved the final manuscript.

\section{Funding}

This work was funded by the intramural research program of the National Library of Medicine of the National Institutes of Health. Funders were not involved in the design of the study and collection, analysis, interpretation of data or writing the manuscript.

\section{Availability of data and materials}

The datasets generated and/or analysed during the current study are available in the geneHummus repository, https://github.com/NCBIHackathons/GeneHummus

\section{Ethics approval and consent to participate}

This article was prepared while Kimberly LeBlanc was a visiting bioinformatician at NCBI. The content is solely the responsibility of the authors and does not necessarily represent the official views of the National Institutes of Health.

\section{Consent for publication}

Not applicable.

\section{Competing interests}

The authors declare that they have no competing interests.

\section{Author details}

'Department of Genetics ETSIAM, University of Córdoba, Córdoba, Spain. ${ }^{2}$ National Center for Biotechnology Information, National Library of Medicine, National Institutes of Health, 8600 Rockville Pike, Bethesda, MD 20894, USA ${ }^{3}$ Department of Biological Sciences, Texas Tech University, TX, Lubbock 79409, USA. ${ }^{4}$ National Institute on Drug Abuse, National Institutes of Health 6001 Executive Blvd, Bethesda, MD 20892, USA. ${ }^{5}$ Department of Computer Science, University of Ibadan, Ibadan, Nigeria.

Received: 30 April 2019 Accepted: 2 July 2019

Published online: 18 July 2019

\section{References}

1. Cook CE, Bergman MT, Finn RD, Cochrane G, Birney E, Apweiler R. The European Bioinformatics Institute in 2016: Data growth and integration. Nucleic Acids Res. 2016;44:D20-6. https://doi.org/10.1093/nar/gkv1352.

2. Fitch WM. Homology a personal view on some of the problems. Trends Genet. 2000;16:227-31

3. Zhao Y. Auxin biosynthesis and its role in plant development. Annu Rev Plant Biol. 2010;61:49-64. https://doi.org/10.1146/annurev-arplant-042 809-112308.

4. Finet C, Berne-Dedieu A, Scutt CP, Marlétaz F. Evolution of the ARF gene family in land plants: old domains, new tricks. Mol Biol Evol. 2013:30:45-56. https://doi.org/10.1093/molbev/mss220.
5. Die JV, Gil J, Millan T. Genome-wide identification of the auxin response factor gene family in Cicer arietinum. BMC Genomics. 2018;19:301. https:// doi.org/10.1186/s12864-018-4695-9.

6. Shen C, Yue R, Sun T, Zhang L, Xu L, Tie S, et al. Genome-wide identification and expression analysis of auxin response factor gene family in Medicago truncatula. Front Plant Sci. 2015;6:73. https://doi.org/10.3389/fpls.2015.00073.

7. Sun Y, Wang C, Wang N, Jiang X, Mao H, Zhu C, et al. Manipulation of Auxin Response Factor 19 affects seed size in the woody perennial Jatropha curcas. Sci Rep. 2017;7:40844. https://doi.org/10.1038/srep40844.

8. Li H, Ran K, Sun Q. Genome-wide identification and expression analysis of peach auxin response factor gene families. J Plant Biochem Biotechnol. 2016;25:349-57. https://doi.org/10.1007/s13562-015-0346-2.

9. Baranwal VK, Negi N, Khurana P. Auxin response factor genes repertoire in mulberry: identification, and structural, functional and evolutionary analyses. Genes (Basel). 2017;8. https://doi.org/10.3390/genes8090202.

10. Kalluri UC, Difazio SP, Brunner AM, Tuskan GA. Genome-wide analysis of Aux/IAA and ARF gene families in Populus trichocarpa. BMC Plant Biol. 2007; 7:59. https://doi.org/10.1186/1471-2229-7-59.

11. Jones B, Frasse P, Olmos E, Zegzouti H, Li ZG, Latché A, et al. Downregulation of DR12, an auxin-response-factor homolog, in the tomato results in a pleiotropic phenotype including dark green and blotchy ripening fruit. Plant J. 2002;32:603-13. https://doi.org/10.1046/j.1365-313X.2 002.01450.x.

12. Liu K, Yuan C, Li H, Lin W, Yang Y, Shen C, et al. Genome-wide identification and characterization of auxin response factor (ARF) family genes related to flower and fruit development in papaya (Carica papaya L.). BMC Genomics. 2015;16:901. https://doi.org/10.1186/s12864-015-2182-0.

13. $\mathrm{Xu} \mathrm{Z}$, Ji A, Song J, Chen S. Genome-wide analysis of auxin response factor gene family members in medicinal model plant Salvia miltiorrhiza. Biol Open. 2016:5:848-57. https://doi.org/10.1242/bio.017178.

14. Varshney RK, Song C, Saxena RK, Azam S, Yu S, Sharpe AG, et al. Draft genome sequence of chickpea (Cicer arietinum) provides a resource for trait improvement. Nat Biotechnol. 2013;31:240-6. https://doi.org/10.1038/nbt.24 91.

15. Altschul SF, Gish W, Miller W, Myers EW, Lipman DJ. Basic local alignment search tool. J Mol Biol. 1990:215:403-10. https://doi.org/10.1016/S0022-2836 (05)80360-2.

16. A Grammar of Data Manipulation [R package dplyr version 0.8.0.1]. https:// cran.r-project.org/web/packages/dplyr/index.html. Accessed 23 Mar 2019.

17. Wickham H. Simple, Consistent Wrappers for Common String Operations [R package stringr version 1.4.0].

18. Winter D. "Entrez" in R [R package rentrez version 1.2.1].

19. Hornik K. The Comprehensive R Archive Network. WIREs Comp Stat. 2012;4: 394-8. https://doi.org/10.1002/wics.1212.

20. geneHummus Github repository. https://raw.githubusercontent.com/NCBIHackathons/GeneHummus/master/tutorial.md. Accessed 9 Apr 2019.

21. Federhen S. The NCBI Taxonomy database. Nucleic Acids Res. 2012; 40(Database issue):D136-43. https://doi.org/10.1093/nar/gkr1178.

22. Kuraku S, Zmasek CM, Nishimura O, Katoh K. aLeaves facilitates on-demand exploration of metazoan gene family trees on MAFFT sequence alignment server with enhanced interactivity. Nucleic Acids Res. 2013;41 Web Server issue:W22-8. https://doi.org/10.1093/nar/gkt389.

23. Katoh K, Rozewicki J, Yamada KD. MAFFT online service: multiple sequence alignment, interactive sequence choice and visualization. Brief Bioinformatics. 2017:bbx108. https://doi.org/10.1093/bib/bbx108.

24. Boratyn GM, Thierry-Mieg J, Thierry-Mieg D, Busby B, Madden TL. Magic BLAST, an accurate DNA and RNA-seq aligner for long and short reads. BioRxiv. 2018. https://doi.org/10.1101/390013.

25. SimpleGeneExpression Github repository. https://github.com/NCBIHackathons/SimpleGeneExpression. Accessed 11 Apr 2019

26. Marchler-Bauer A, Bo Y, Han L, He J, Lanczycki CJ, Lu S, et al. CDD/ SPARCLE: functional classification of proteins via subfamily domain architectures. Nucleic Acids Res. 2017;45:D200-3. https://doi.org/10.1093/ nar/gkw1129.

27. O'Leary NA, Wright MW, Brister JR, Ciufo S, Haddad D, McVeigh R, et al. Reference sequence (RefSeq) database at NCBI: current status, taxonomic expansion, and functional annotation. Nucleic Acids Res. 2016;44:D733-45. https://doi.org/10.1093/nar/gkv1189.

28. Hane JK, Ming Y, Kamphuis LG, Nelson MN, Garg G, Atkins CA, et al. A comprehensive draft genome sequence for lupin (Lupinus angustifolius), an emerging health food: insights into plant-microbe interactions and legume 
evolution. Plant Biotechnol J. 2017;15:318-30. https://doi.org/10.1111/ pbi.12615.

29. Ha CV, Le DT, Nishiyama R, Watanabe Y, Sulieman S, Tran UT, et al. The auxin response factor transcription factor family in soybean: genome-wide identification and expression analyses during development and water stress. DNA Res. 2013;20:511-24. https://doi.org/10.1093/dnares/dst027.

30. Shiny app of ARFs in legume species. https://genehummus.shinyapps.io/ testshiny/. Accessed 11 Apr 2019

31. Shiny app Github repository. https://raw.githubusercontent.com/NCBIHackathons/GeneHummus/R_package/Shiny.R. Accessed 11 Apr 2019.

32. Okushima Y, Overvoorde PJ, Arima K, Alonso JM, Chan A, Chang C, et al. Functional genomic analysis of the AUXIN RESPONSE FACTOR gene family members in Arabidopsis thaliana: unique and overlapping functions of ARF7 and ARF19. Plant Cell. 2005;17:444-63. https://doi.org/10.1105/tpc.104. 028316

33. Yu H, Soler M, Mila I, San Clemente H, Savelli B, Dunand C, et al. Genomewide characterization and expression profiling of the AUXIN RESPONSE FACTOR (ARF) gene family in Eucalyptus grandis. PLoS ONE. 2014;9:e108906. https://doi.org/10.1371/journal.pone.0108906.

34. Mun J-H, Yu H-J, Shin JY, Oh M, Hwang H-J, Chung H. Auxin response factor gene family in Brassica rapa: genomic organization, divergence, expression, and evolution. Mol Genet Genomics. 2012;287:765-84. https://doi.org/10.1 007/s00438-012-0718-4.

35. Kumar R, Tyagi AK, Sharma AK. Genome-wide analysis of auxin response factor (ARF) gene family from tomato and analysis of their role in flower and fruit development. Mol Genet Genomics. 2011;285:245-60. https://doi. org/10.1007/s00438-011-0602-7.

36. Wan S, Li W, Zhu Y, Liu Z, Huang W, Zhan J. Genome-wide identification, characterization and expression analysis of the auxin response factor gene family in Vitis vinifera. Plant Cell Rep. 2014;33:1365-75. https://doi.org/10.1 007/s00299-014-1622-7.

37. NCBI Resource Coordinators. Database resources of the National Center for Biotechnology Information. Nucleic Acids Res. 2016;44:D7-19. https://doi. org/10.1093/nar/gkv1290

38. Chen X, Zheng J, Fu Z, Nan P, Zhong Y, Lonardi S, et al. Assignment of orthologous genes via genome rearrangement. IEEE/ACM Trans Comput Biol Bioinform. 2005:2:302-15. https://doi.org/10.1109/TCBB.2005.48.

39. Shi G, Peng M-C, Jiang T. MultiMSOAR 2.0: an accurate tool to identify ortholog groups among multiple genomes. PLOS ONE. 2011;6:e20892. https://doi.org/10.1371/journal.pone.0020892.

40. Li L, Stoeckert CJ, Roos DS. OrthoMCL: identification of ortholog groups for eukaryotic genomes. Genome Res. 2003;13:2178-89. https://doi.org/10.1101/ gr.1224503.

41. Fischer S, Brunk BP, Chen F, Gao X, Harb OS, lodice JB, et al. Using OrthoMCL to assign proteins to OrthoMCL-DB groups or to cluster proteomes into new ortholog groups. Curr Protoc Bioinformatics. 2011; Chapter 6:Unit 6:12.1-19. https://doi.org/10.1002/0471250953.bi0612s35.

42. Kersey PJ, Allen JE, Allot A, Barba M, Boddu S, Bolt BJ, et al. Ensembl Genomes 2018: an integrated omics infrastructure for non-vertebrate species. Nucleic Acids Res. 2018:46:D802-8. https://doi.org/10.1093/nar/gkx1011.

43. Li Z, Baniaga AE, Sessa EB, Scascitelli M, Graham SW, Rieseberg LH, et al. Early genome duplications in conifers and other seed plants. Sci Adv. 2015; 1:e1501084. https://doi.org/10.1126/sciadv.1501084

44. Bertioli DJ, Cannon SB, Froenicke L, Huang G, Farmer AD, Cannon EKS, et al. The genome sequences of Arachis duranensis and Arachis ipaensis, the diploid ancestors of cultivated peanut. Nat Genet. 2016;48:438-46. https:// doi.org/10.1038/ng.3517.

\section{Publisher's Note}

Springer Nature remains neutral with regard to jurisdictional claims in published maps and institutional affiliations.

Ready to submit your research? Choose BMC and benefit from:

- fast, convenient online submission

- thorough peer review by experienced researchers in your field

- rapid publication on acceptance

- support for research data, including large and complex data types

- gold Open Access which fosters wider collaboration and increased citations

- maximum visibility for your research: over $100 \mathrm{M}$ website views per year

At BMC, research is always in progress.

Learn more biomedcentral.com/submissions 